\title{
REGISTROS NOTABLES DE MAMÍFEROS EN EL SUR DEL DISTRITO FEDERAL, MÉXICO
}

\author{
HORACIO V.BÁRCENAS Y RODRIGO A. MEDELLÍN \\ Instituto de Ecología, Universidad Nacional Autónoma de México, \\ Apartado Postal 70-275, 04510 México D. F., MEXICO. \\ correo electrónico: hbarcenas@miranda.ecologia.unam.mx
}

\begin{abstract}
The aim of this note is to report current records of mammals using camera-trap and other evidences at the south of Mexico City. The survey periods were carried out between march 2006 to June 2007. Total of 16 species in 10 families of mammals were recorded.
\end{abstract}

Key words: Records, mammals, Tlalpan, Distrito Federal, Mexico.

Palabras clave: Registros, mamíferos, Tlalpan, Distrito Federal, México.

De todas las regiones biológicas en las que se divide México, el Eje Neovolcánico Transversal tiene una alta diversidad y número de especies endémicas (Ceballos et al., 2005). El área es muy importante por que en ella convergen dos regiones biogeográficas Neártica y Neotropical. El Eje Neovolcánico Transversal atraviesa nuestro país de costa a costa por su parte central, desde el Golfo de México hasta el Océano Pacifico (Romero y Velázquez, 1999). También atraviesa al sur la cuenca de México en los estados de Morelos, Estado de México y Distrito Federal. Esta región provee invaluables beneficios a la población de la ciudad de México y al Área Metropolitana, ya que a través de ella se recargan los acuíferos de los que depende el abastecimiento de agua de millones de habitantes de la ciudad y representa la principal área de captura de bióxido de carbono y suministro de oxígeno para el Valle de México. En esta zona se han registrado 59 especies de mamíferos silvestres, 40 de los cuales tienen afinidad Neártica y 19 Neotropical (Monroy-Vilchis et al., 1999). Los principales tipos de vegetación en el sur de la cuenca del valle de México son bosque de coníferas, bosque de encino, bosque mixto y pastizal, (Arriaga et al., 2000).

Durante el desarrollo del estudio "Estimación de la densidad poblacional y dieta del lince (Lynx rufus) en Aguascalientes y el Distrito Federal, México", en la localidad de San Miguel Topilejo en la Delegación Tlalpan, a 20 kilómetros al sur de la Ciudad de México, se registraron por medio de cámaras-trampa varios mamíferos además del lince. El propósito de este trabajo es reportar estos registros recientes (2006-2007). 
En varios casos consideramos particularmente relevante reportar la presencia de esas especies, por la escasa información que existe sobre ellos. Todas las fotos fueron tomadas con un equipo de fototrampeo Cam Trakker y Stealth Cam cuyo sistema infrarrojo pasivo es activado por calor y movimiento. Dicho sensor está conectado a una cámara en la que se utilizaron rollos de 36 exposiciones, asa 200 a color.

Sylvilagus floridanus (J. A. Allen, 1890)

Este conejo es muy abundante en la región, en zonas con perturbaciones antropogénicas (Angermann et al., 1990). Se registró el día 10 junio del 2007 a las 08:56 h en una estación de trampeo ubicada en el pastizal en las coordenadas $19^{\circ} 05^{\prime} 59.75 \mathrm{~N}$, 9909`59.10 O, a una altitud de $2856 \mathrm{msnm}$ en una brecha de $50 \mathrm{~cm}$ de ancho.

\section{Conepatus leuconotus (Lichtenstein, 1832)}

Este zorrillo de espalda blanca es el más grande de las especies que se distribuyen en nuestro país. Hasta hace poco tiempo C. leuconotus y C. mesoleucus se consideraban especies diferentes, pero recientemente Dragoo et al. (2003) evaluaron la variación morfométrica y el ADN mitocondrial concluyendo que se trata de una sola especie. Nuestro registro se ubica a 12 km del reportado por Ramírez-Pulido (1971) en Huitzilac, Morelos a $3200 \mathrm{msnm}$. Ambas localidades son notables ya que la mayoría de sus registros provienen de altitudes mucho menores, en zonas tropicales. Registramos a este zorrillo el 12 de junio del 2007 a las 01:32 h en la misma estación en la que detectamos a $S$. floridanus.

Mephitis macroura (Lichtenstein, 1832)

El zorrillo listado es reportado como abundante en la zona y es una especie beneficiada por las actividades antropogénicas (Mendoza y Ceballos, 2005; Monroy-Vilchis et al., 1999). Este zorrillo fue fototrampeado el día 8 junio del 2007 a las $04: 41$ h en un camino de terracería ubicado a $400 \mathrm{~m}$ de la caseta de vigilancia de CORENA conocida como "La Paloma", en las coordenadas 1905’39.69" N, 99¹048.78" O, 2745 msnm.

Mustela frenata (Lichtenstein, 1832)

La comadreja es un carnívoro conocido en esta zona con afinidad Neártica (MonrroyVilchis et al., 1999). Nosotros registramos a un individuo el día 5 de junio del 2007 a las 18:47 h en las coordenadas $19^{\circ} 05^{\prime} 37.43^{\prime \prime} \mathrm{N}, 99^{\circ} 10^{\prime} 30.12^{\prime \prime} \mathrm{O}, 2960$ msnm en un camino de 
terracería donde posteriormente se confirmo visualmente su presencia el día 13 de junio del 2007 a las 11:30h.

Procyon lotor (Linnaeus, 1758)

El mapache se fototrampeó el día 10 de abril del 2006 a las 21:45 h en un camino de terracería. La estación de muestreo se ubicó a tan solo 5 metros de la autopista MéxicoCuernavaca y a $10 \mathrm{~m}$ del borde del bosque en las coordenadas $19^{\circ} 06^{\prime} 50.09 " \mathrm{~N}$, $991^{\circ} 10^{\prime} 57.09^{\prime \prime} \mathrm{O}$, a una altitud de $2750 \mathrm{msnm}$. Esta especie se ha adaptado a vivir en una gran variedad de hábitats, siempre y cuando existan cuerpos de agua permanente. Sin embargo, en la zona de estudio el cuerpo de agua artificial más cercano se encuentra a aproximadamente a $5 \mathrm{~km}$ en línea recta y no tiene agua disponible todo el año. Se ha reportado que la mayor distancia recorrida en una noche es de $1.9 \mathrm{~km}$ y que en promedio es de $0.4 \mathrm{~km}$ (Lotze y Anderson, 1979). Probablemente este reporte se encuentra en el extremo altitudinal de la distribución de esta especie en México (Hall, 1981; López-Wilchis y López-Jardines, 1998).

Canis latrans (Say, 1823)

Este cánido ya ha sido reportado de la región (Monroy-Vilchis et al., 1999). Registramos a la especie el día 3 de junio del 2007 a las 23:19 h en una brecha al borde del Volcán Chichinautzin en las coordenadas 19061․ 18 "N, 9909`38.69" O. 2750 msnm.

Lynx rufus (Schreber, 1777)

De este felino tenemos 10 registros en el área muestreada. La foto que se presenta en este trabajo fue tomada el día 12 de abril del 2006 a las 20:42 h en la base del volcán conocido como Hoyo en las coordenadas 1905’20.93 N, 99¹0’16.14"O, 3072 msnm (Figura 1). La mitad de este terreno pertenece al área Comunal de San Miguel Topilejo, D. F. y la otra mitad al Municipio de Coajomulco, Morelos. Es muy relevante que el lince continúe estando presente en números relativamente importantes en una zona tan cercana a la Ciudad de México y que tiene alteraciones tan importantes como la región de Parres (Bárcenas y Medellín, 2007).

Odocoileus virginianus (Zimmermann, 1780)

Desde las primeras vistas al área de muestreo en el mes de marzo del 2005 se identificaron huellas de esta especie, sin embargo fue hasta el día 7 junio del 2007 a las 21:56 h cuando se registró a la especie mediante fototrampeo en las coordenadas $19^{\circ} 05^{\prime} 20.14^{\prime \prime} \mathrm{N}, 99^{\circ} 10^{\prime} 48.24^{\prime \prime}$ O, a 3182 msnm (Figura 2) y posteriormente el día 10 de 


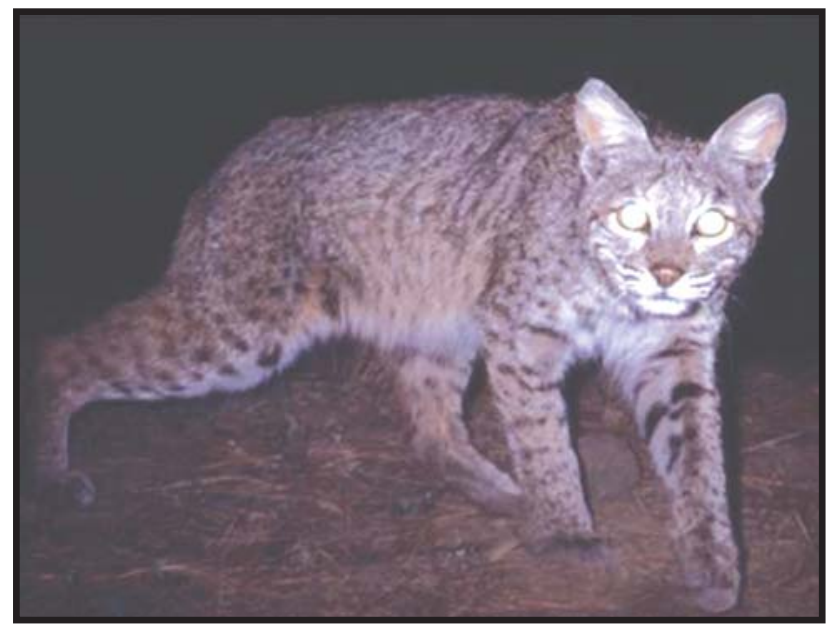

Figura 1. Lynx rufus. Registro obtenido por medio de trampas fotográficas en el Área Comunal de San Miguel Topilejo, en la delegación Tlalpan, Distrito Federal.

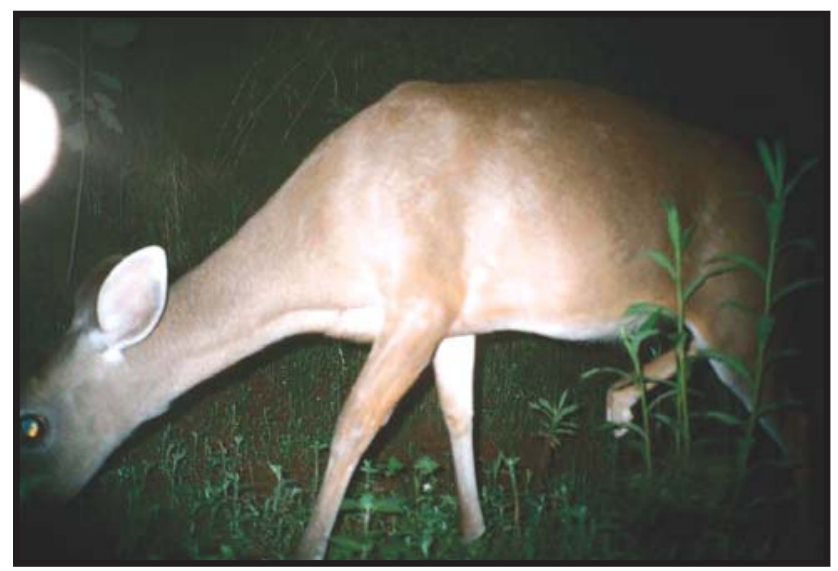

Figura 2. Odocoileus virginianus. Desde hace más de 50 años no se había registrado esta especie dentro de los límites del Distrito Federal. 
octubre del 2007 a las 09:45 h en las coordenadas $19^{\circ} 05^{\prime} 24.62^{\prime \prime} \mathrm{N}, 99^{\circ} 11^{\prime} 05.60^{\prime \prime} 0$, a una altitud de $3053 \mathrm{msnm}$. Actualmente contamos con 2 registros fotográficos y con 14 registros de huellas en esta área. Dado que es una especie que sufre de una fuerte presión de cacería, es muy alentador encontrar que todavía existen individuos que pueden representar una alternativa para recuperar sus poblaciones.

\section{DISCUSIÓN Y CONCLUSIÓN}

Consideramos que los hallazgos de especies como las reportadas aquí en condiciones de alteración importantes, representan una oportunidad de iniciar a acciones de conservación y recuperación de la biodiversidad en la zona. Nuestros registros muestran que esta región mantiene aún a los mamíferos medianos y grandes reportados hace 54 años (Villa-Ramírez, 1953). En el caso particular del mapache ( $P$. lotor) el registro más reciente era de 1931 (Helgen y Wilson, 2005) y en el caso del venado cola blanca (O. virginianus) el primer registro dentro del Distrito Federal fue el descrito por Herrera en 1890 (Ceballos y Galindo, 1984), y el más reciente es de hace 54 años. Sin embargo Villa-Ramírez (1953) consideró que el ejemplar colectado en 1950 no era residente si no más bien emigrante y que provenía de zonas más alejadas del Estado de México. En el caso de este estudio contamos con evidencia continua (20052007) de su presencia en el área de estudio. Algunas otras especies que hemos documentado a través de sus huellas u otra evidencia son el meteorito (Microtus mexicanus), la rata magueyera (Neotoma mexicana), el ratón de los volcanes (Neotomodon alstoni), el teporingo (Romerolagus diazi), el conejo montés (Sylvilagus cunicularius), el armadillo (Dasypus novemcinctus), el ardillón (Spermophilus variegatus) y la ardilla gris (Sciurus aureogaster). La permanencia de estas especies en la zona debe ser asegurada en el corto plazo. Las autoridades del Distrito Federal y de la Delegación Tlalpan corren con la responsabilidad de impedir su extirpación y promover y asegurar su recuperación para garantizar su permanencia en la zona para las generaciones futuras. Un programa de conservación para esta región debe contar con el respaldo y la participación de la comunidad académica y de los pobladores locales.

\section{AGRADECIMIENTOS}

Agradecemos al Laboratorio de Conservación y Ecología de Vertebrados Terrestres del Instituto de Ecología, de la UNAM, CONABIO, Bioconciencia y a la Association of Fish and Wildlife Agencies y Wildlife Trust Alliance por los fondos proporcionados, a E. Nájera, A. Rosas y a S. Romo por su ayuda en el trabajo de campo, a G. López por la revisión del manuscrito y a J. Cruzado por la ayuda en la identificación de los 
roedores. A la brigada "Nogal 23" de la Regional 2 de CORENA y al Sr. Aniceto Ruiz, Presidente de los Bienes Comunales del Área Comunal de San Miguel Topilejo.

\section{LITERATURACITADA}

Angermann, R., J.E.C. Flux, J.A. Chapman y A.T. Smith. 1990. Lagomorph classification. Pp. 7-13, en: Rabbits, Hares and Pikas. Status Survey and Conservation Action Plan (J.A. Chapman y J.E.C. Flux, eds.). International Union for Conservation of Nature and Natural Resources, Gland, Suiza.

Arriaga, L., J.M. Espinoza, C. Aguilar, M. Martínez, L. Gómez y E. Loa. 2000. Regiones Terrestres Prioritarias de México. CONABIO. México, D.F.

Bárcenas, V.H. y R.A. Medellín. 2007. Estimación de la densidad y dieta del lince en San Miguel Topilejo, Distrito Federal y Sierra Seri, Aguascalientes. 2do Reporte Técnico. CONABIO.

Ceballos, G., J. Arroyo-Cabrales, R.A. Medellín, G.L. Medrano, y G. Oliva 2005. Diversidad y Conservación de los Mamíferos de México. Pp. 21-66, en: Los Mamíferos Silvestres de México (G. Ceballos. y G. Oliva, eds.). Fondo de Cultura Económica y CONABIO. México, D.F.

Ceballos, G. y C. Galindo. 1984. Mamíferos silvestres de la cuenca de México. LimusaInstituto de Ecología. México, D.F.

Dragoo, J.W., R.L. Honeycutt, y D.J. Schmidly. 2003. Taxonomic status of white-backed hognosed skunks, genus Conepatus (Carnivora: Mephitidae). Journal of Mammalogy, 84:159176.

Hall, E.R. 1981. The mammals of North America. Segunda Edición. John Wiley and Sons, New York.

Helgen, K.M. y D.E. Wilson. 2005. A systematic and zoogeographic overview of the ranccoons of Mexico and Central America. Pp. 221-236, en: Contribuciones Mastozoológicas en homenaje a Bernardo Villa (V. Sánchez-Cordero y R.A. Medellín, eds.). Instituto de Biología, UNAM; Instituto de Ecología, UNAM; CONABIO. México, D.F.

López-Wilchis, R. y J. López-Jardines. 1998. Los Mamíferos de México depositados en colecciones de Estados Unidos y Canadá. Universidad Autónoma Metropolitana Unidad Iztapalapa. Universidad Autónoma Metropolitana. México, D.F.

Lotze, J.-H. y S. Anderson. 1979. Procyon lotor. Mammalian Species, 119:1-8.

Mendoza, D.A. y G. Ceballos. 2005. Conepatus leuconotus. Pp. 386-387, en: Los Mamíferos Silvestres de México (Ceballos, G. y G. Oliva, eds.). Fondo de Cultura Económica y CONABIO. México, D.F.

Monrroy-Vilchis, O., H. Rangel-Cordero, M. Aranda, A. Velázquez y J.F. Romero. 1999. Los mamíferos de hábitat templado del sur de la cuenca de México. Pp. 141-159, en: La Biodiversidad de la Región de Montaña del sur de la Cuenca de México. (Romero, J.F.y A. Velázquez, eds.). Universidad Autónoma Metropolitana. México.

Ramírez-Pulido, J. 1971. Contribución al estudio de mamíferos del Parque Nacional "Lagunas de Zempoala", Morelos, México. Anales del Instituto de Biología, UNAM, 40:253-290. 
Romero, J.F. y A. Velázquez. 1999. La región de montaña del sur de la Cuenca de México: una revisión de su importancia biológica, en: La Biodiversidad de la Región de Montaña del sur de la Cuenca de México. (Romero, J.F. y A. Velázquez, eds.). Universidad Autónoma Metropolitana. México. D.F.

Villa-Ramírez, B. 1953. Mamíferos silvestres del Valle de México. Anales del Instituto de Biología, UNAM, 23:269-492. 